\title{
Fidelity measure and conservation of information in general probabilistic theories
}

\author{
C. ZAnder ${ }^{1}$ and A. R. Plastino ${ }^{1,2,3(a)}$ \\ ${ }^{1}$ Department of Physics, University of Pretoria - 0002 Pretoria, South Africa \\ ${ }^{2}$ National University La Plata, UNLP-CREG-CONICET - C.C. 727, 1900 La Plata, Argentina \\ ${ }^{3}$ Instituto Carlos I de Física Teórica y Computacional, University of Granada - Granada, Spain, EU
}

received 12 January 2009; accepted in final form 16 March 2009

published online 21 April 2009

PACS 89.70.-a - Information and communication theory

PACS 05.90.+m - Other topics in statistical physics, thermodynamics, and nonlinear dynamical systems

PACS 03.65.Ta - Foundations of quantum mechanics; measurement theory

\begin{abstract}
We investigate the main features of a measure of fidelity between states in a general family of probabilistic theories admitting classical probability theory and standard quantum theory as particular instances. We apply the aforementioned measure to investigate informationtheoretical features of these theories related to the conservation of information during the evolution of closed physical systems. In particular, we derive a generalization of a fundamental result in quantum theory relevant for the measurement problem: Zurek's recent extension of the no-cloning theorem.
\end{abstract}

Copyright (C) EPLA, 2009

Introduction. - The physics of information and computation [1-6] has been the focus of an intense and increasing research activity in recent years [5-13]. Part of this research effort has been devoted to determine the ultimate limits imposed by the fundamental laws of physics on any device processing or transmitting information [6-8]. On the other hand, a growing body of theoretical developments indicate that the concept of information constitutes an essential ingredient for a deep understanding of physical systems and processes [1-6,9-12]. Ideas, techniques, and models inspired by the theoretical analysis of information processing devices proved to be relevant for the study of a variegated range of physical scenarios, including applications to subjects as diverse as quantum thermodynamical machines [9] or the fundamental limits on the accuracy of spacetime measurements [10]. Interesting attempts have been recently made to derive the basic formalism of quantum theory from information-theoretical concepts [11]. The advent of quantum information science, and the concomitant discovery of the novel, subtle, and counter-intuitive ways of processing and transmitting information allowed by quantum mechanics $[6,13]$ greatly stimulated the interest in these lines of enquiry. Work on quantum information

\footnotetext{
${ }^{(a)}$ E-mail: arplastino@maple.up.ac.za
}

theory is shedding new light on the foundations of quantum theory and on the relationships between the classical and quantum mechanical descriptions of Nature. An interesting and powerful new approach to these issues has been recently advanced by Barnum, Barrett, Leifer, and Wilce [14] (from here on BBLW). These researchers proposed to investigate systematically the informationtheoretical aspects of probabilistic descriptions of Nature, within a general operational framework that encompasses classical probabilities and quantum mechanical probabilities as two particular instances of a wide family of probabilistic theories. Similar or related ideas have been discussed previously in the literature (see $[15,16]$ and refs. in [14]), but BBLW provided the first systematic analysis of fundamental aspects of information physics, such as the no-broadcasting principle, within the general framework.

There is much to be gained from exploring generalizations of existing physical theories [17]. Historically, this has been wonderfully illustrated by the great stimulus that research on general relativity got from the study of alternative theories of gravitation [18]. This kind of approach allows for the identification and investigation of important problems that otherwise might have gone unnoticed. For example, generalizations of the standard quantum theory are currently being used to study one of the most 
intriguing open questions on the foundations of quantum mechanics: Why does Nature not permit the maximum amount of non-locality consistent with causality? [19]. Last, but certainly not least, these lines of research may shed new light on the epistemic vs. ontic debate on the basic meaning of the quantum mechanical formalism [20].

The aim of this work is to investigate the main properties of a measure of fidelity between states in general probabilistic theories described by the BBLW framework, and to explore its application to the study of basic informationrelated processes. We shall apply the alluded fidelity measure to derive, within the aforementioned probabilistic theories, a generalization of a basic result in quantum theory with implications for the measurement problem: Zurek's recent extension of the no-cloning theorem.

The BBLW operational framework. - The basic ingredients of the BBLW approach are the concepts of states, effects, measurements, and transformations, as defined below (see [14] for details). These concepts generalize some well-known concepts of standard quantum mechanics.

States. The set $\Omega$ of all possible states of a physical system is a finite-dimensional, compact, and convex set. The extreme points of the set $\Omega$ are called "pure" states. In standard quantum mechanics the state set $\Omega$ corresponds to the set of all statistical operators $\rho$ on the system's Hilbert space $\mathcal{H}$. In classical mechanics, the set of states is given by the set of all normalized probability distributions defined on an appropriate, classical phase space. A state determines the probabilities for different measurement outcomes. Let $e(\omega)$ denote the probability of obtaining outcome $e$ when the system is in state $\omega$.

Effects. Mathematically, the probability $e(\omega)$ is given by an affine functional $e: \Omega \rightarrow[0,1]$, which is referred to as an effect. In particular, we have the unit effect $u$ verifying $u(\omega)=1$ for all $\omega \in \Omega$. In quantum theory, an effect is represented by a positive operator $E$ bounded by 0 and the identity operator $I$. The probability of the concomitant outcome (if the system is in state $\rho$ ) is $\operatorname{Tr}(E \rho)$. The unit effect is given by the identity $I$. We are going to assume that a state $\omega$ is completely characterized by the probabilities $e(\omega)$. In other words, two states $\omega_{1}$ and $\omega_{2}$ yield the same probabilities for all effects $e$ (i.e., $e\left(\omega_{1}\right)=e\left(\omega_{2}\right)$ for all $\left.e\right)$ iff $\omega_{1}=\omega_{2}$.

Measurement. A measurement is identified with a set of effects $\left\{e_{i}\right\}$ such that $\sum_{i} e_{i}=u$ and consequently, $\sum_{i} e_{i}(\omega)=1$ for all $\omega \in \Omega$. This generalizes the concept of a POVM in quantum theory.

Transformations. Physical transformations of a system are described by an appropriate set of affine mappings $T: \Omega \rightarrow \Omega^{\prime}$, where $\Omega$ and $\Omega^{\prime}$ stand, respectively, for the system's state space before and after the transformation. These transformations play the role of linear, trace-preserving, completely positive maps in the standard quantum formalism.
The rules for the description of bipartite systems constitute another important component of the BBLW framework.

Bipartite Systems. Let us consider a bipartite system $A B$ constituted by subsystems $A$ and $B$. Lets denote the state spaces of subsystems $A$ and $B$, respectively, by $\Omega_{A}$ and $\Omega_{B}$. The state space of the composite system $A B$ is denoted by $\Omega_{A B}$. A joint state $\omega_{A B} \in \Omega_{A B}$ specifies completely the joint probabilities $\omega_{A B}\left(e_{A}, e_{B}\right)$ corresponding to pairs of effects $\left(e_{A}, e_{B}\right)$, where $e_{A, B}$ are effects defined with respect to the individual subsystems $A$ and $B$. Conversely, the joint probabilities for all pairs of effects $\left(e_{A}, e_{B}\right)$ fully characterize the joint state $\omega_{A B}$. The joint probabilities comply with the no-signaling constraint.

For each state $\omega_{A B}$ of the bipartite system there are reduced states $\omega_{A, B}$ characterized by $e_{A}\left(\omega_{A}\right)=\omega_{A B}\left(e_{A}\right.$, $\left.u_{B}\right)$ and $e_{B}\left(\omega_{B}\right)=\omega_{A B}\left(u_{A}, e_{B}\right)$. If either marginal state is pure, then the joint state is factorizable: $\omega_{A B}=\omega_{A} \otimes \omega_{B}$ and $\omega_{A B}\left(e_{A}, e_{B}\right)=e_{A}\left(\omega_{A}\right) e_{B}\left(\omega_{B}\right)$.

Time evolution of closed systems and measurements as physical processes. - We are going to assume that there is a set of invertible transformations $\Gamma: \Omega \rightarrow \Omega$ representing the fundamental transformations that a closed physical system (with state space $\Omega$ ) can experience. Each one of these transformations $\Gamma$ admits an inverse $\Gamma^{(-1)}$, which is also a legitimate physical transformation for the system under consideration. For all $\omega \in \Omega$, we have $\Gamma^{(-1)}(\Gamma(\omega))=\Gamma\left(\Gamma^{(-1)}(\omega)\right)=\omega$.

These invertible transformations are to be regarded as the truly fundamental ones. We assume that when one studies a given physical system it is always possible to extend it, and regard it as a subsystem of a larger, closed system. In this way, all possible transformations affecting the original system are manifestations of invertible transformations acting upon the extended closed system. This assumption is not part of the BBLW framework as exposed in [14]. However, both classical and quantum mechanics comply with it and it is, therefore, reasonable to regard it as a basic ingredient of our present understanding of the fundamental laws of physics.

Since measurement processes are themselves physical processes, there has to be a physical consistency relation between the set of all possible measurements and the set of all invertible operations acting upon a physical system. If we first apply a transformation $\Gamma$ to our system, and then perform a measurement $\left\{e_{i}\right\}$ upon it, the overall process can always be regarded as performing a certain measurement $\left\{\tilde{e}_{i}\right\}$ on the initial state of the system. For instance, if we consider the state of a spin-(1/2) particle in standard quantum mechanics, to first apply a $\pi / 2$ rotation around the $x$-axis and then measure $S_{z}$ is equivalent to measuring $S_{y}$ on the original state. In the context of a general probabilistic theory, given an effect $e$ and an invertible operation $\Gamma$ there exists an effect $\tilde{e}$ such that, for all states $\omega \in \Omega, \tilde{e}(\omega)=e(\Gamma(\omega))$. As a consequence, given a measurement $\left\{e_{i}\right\}$ and an invertible 
transformation $\Gamma$ there is another measurement $\left\{\tilde{e}_{i}\right\}$ such that $\tilde{e}_{i}(\omega)=e_{i}(\Gamma(\omega))$ for all $\omega \in \Omega$.

Fidelity measure for states in general probabilistic theories. - As a generalized fidelity measure between two states $\omega_{1}$ and $\omega_{2}$, we propose the infimum (i.e., the largest lower bound) of the set of overlap values between the pairs of probability distributions $e_{i}\left(\omega_{1,2}\right)$ associated with all the possible measurements $\left\{e_{i}\right\}$,

$$
\mathcal{F}\left[\omega_{1}, \omega_{2}\right]=\inf _{\left\{e_{i}\right\}} \sum_{i} \sqrt{e_{i}\left(\omega_{1}\right) e_{i}\left(\omega_{2}\right)} .
$$

The alluded set of overlap values is bounded from below (all the overlaps are non-negative numbers) and consequently the aforementioned infimum always exists and we have $0 \leqslant \mathcal{F}\left[\omega_{1}, \omega_{2}\right] \leqslant 1$, with $\mathcal{F}\left[\omega_{1}, \omega_{2}\right]=1$ iff $\omega_{1}=\omega_{2}$. The fidelity $\mathcal{F}\left[\omega_{1}, \omega_{2}\right]$ measures how well the two states $\omega_{1}$ and $\omega_{2}$ can be distinguished by recourse to measurements. The extreme case $\mathcal{F}\left[\omega_{1}, \omega_{2}\right]=0$ corresponds to a pair of states that can be distinguished with certainty by recourse to an appropriate measurement, while in the other limit case $\mathcal{F}\left[\omega_{1}, \omega_{2}\right]=1$ the two states are identical and consequently indistinguishable. It is worth emphasizing that eq. (1) provides an operational definition of the fidelity $\mathcal{F}\left[\omega_{1}, \omega_{2}\right]$ since the quantities appearing in the right-hand side of (1) can be determined experimentally.

In the particular case of standard quantum mechanics the measure (1) reduces to the fidelity measure between density matrices [13]

$$
\mathcal{F}[\rho, \sigma]=\operatorname{Tr} \sqrt{\rho^{1 / 2} \sigma \rho^{1 / 2}} .
$$

Now we are going to study the main properties of the generalized fidelity functional (1). As we are going to see, most of the properties exhibited by the quantum mechanical fidelity (2) are shared by the measure (1), and can be proved in general, without using specific features of the quantum mechanical formalism.

Given a pair of states $\omega_{1,2}$ let us consider the two states $\omega_{1,2} \otimes \omega_{0}$, where $\omega_{0}$ is a state of an ancilla system. Any measurement done on $\omega_{i} \otimes \omega_{0}$ can be construed as a measurement on $\omega_{i}$ (the state $\omega_{0}$ of the ancilla system can be regarded as characterizing the initial setting of (part of) the measurement apparatus). Indeed, the act of performing a measurement on $\omega_{i} \otimes \omega_{0}$ can be interpreted as follows: Take the system to be measured (which is in the unknown state $\omega_{i}$ ), "attach" to it an ancilla system in a standard initial state $\omega_{0}$, and perform a measurement on the resulting composite (which is in state $\omega_{i} \otimes \omega_{0}$ ). Physically, it is clear that this procedure is tantamount to performing a measurement on $\omega_{i}$. In point of fact, in any real measurement one always has to "attach" ancilla systems (in prescribed, standard initial states) to the system being measured. Now, since the set of possible measurements on $\omega_{0} \otimes \omega_{i}$ is a subset of all the possible measurements on $\omega_{i}$, it follows from (1) that $\mathcal{F}\left[\omega_{1} \otimes \omega_{0}\right.$, $\left.\omega_{2} \otimes \omega_{0}\right] \geqslant \mathcal{F}\left[\omega_{1}, \omega_{2}\right]$. Conversely, a measurement on $\omega_{i}$ can also be regarded as a measurement performed on $\omega_{i} \otimes \omega_{0}$. Therefore, (1) implies that $\mathcal{F}\left[\omega_{1} \otimes \omega_{0}, \omega_{2} \otimes \omega_{0}\right] \leqslant$ $\mathcal{F}\left[\omega_{1}, \omega_{2}\right]$ and, consequently,

$$
\mathcal{F}\left[\omega_{1} \otimes \omega_{0}, \omega_{2} \otimes \omega_{0}\right]=\mathcal{F}\left[\omega_{1}, \omega_{2}\right] .
$$

The argument leading to the inequality $\mathcal{F}\left[\omega_{1} \otimes \omega_{0}, \omega_{2} \otimes\right.$ $\left.\omega_{0}\right] \geqslant \mathcal{F}\left[\omega_{1}, \omega_{2}\right]$ does not constitute a formal mathematical proof. In the strict mathematical sense, this inequality has to be regarded as an assumption that we are making about the behavior of the fidelity function. But it is an assumption based on a compelling physical argument. The second inequality leading to (3) can be obtained as a particular case of (5), which is proved below.

Given a pair of joint states $\omega_{1,2}^{(A B)}$ (of a bipartite system $A B)$ with reduced states $\omega_{1,2}^{(A)}$ and $\omega_{1,2}^{(B)}$, we have

$$
\mathcal{F}\left[\omega_{1}^{(A)}, \omega_{2}^{(A)}\right] \geqslant \mathcal{F}\left[\omega_{1}^{(A B)}, \omega_{2}^{(A B)}\right]
$$

since any measurement performed on subsystem $A$ can be construed as a measurement upon the composite system $A B$.

Let us consider now the fidelity $\mathcal{F}\left[\omega_{1}^{(A)} \otimes \omega_{1}^{(B)}, \omega_{2}^{(A)} \otimes\right.$ $\left.\omega_{2}^{(B)}\right]$ between two factorizable states. If one uses eq. (1) to compute $\mathcal{F}\left[\omega_{1}^{(A)} \otimes \omega_{1}^{(B)}, \omega_{2}^{(A)} \otimes \omega_{2}^{(B)}\right]$ it is clear that evaluating the infimum over the restricted family of product measurements $\left\{\left(e_{A i}, e_{B i}\right)\right\}$ (which would yield $\left.\mathcal{F}\left[\omega_{1}^{(A)}, \omega_{2}^{(A)}\right] \mathcal{F}\left[\omega_{1}^{(B)}, \omega_{2}^{(B)}\right]\right)$ cannot lead to a larger number than the one obtained by an unrestricted evaluation of the infimum. Consequently, we have

$$
\begin{aligned}
\mathcal{F} & {\left[\omega_{1}^{(A)} \otimes \omega_{1}^{(B)}, \omega_{2}^{(A)} \otimes \omega_{2}^{(B)}\right] \leqslant } \\
& \mathcal{F}\left[\omega_{1}^{(A)}, \omega_{2}^{(A)}\right] \mathcal{F}\left[\omega_{1}^{(B)}, \omega_{2}^{(B)}\right] .
\end{aligned}
$$

Given two sets of states $\left\{\omega_{1 i}, i=1, \ldots, M\right\}$ and $\left\{\omega_{2 i}, i=\right.$ $1, \ldots, M\}$ of a given system, and a measurement $\left\{e_{j}\right\}$, let us consider the fidelity measure between two convex linear combinations $\sum_{i} p_{i} \omega_{1 i}$ and $\sum_{i} q_{i} \omega_{2 i}$ of the alluded states, where $\left\{p_{i}, i=1, \ldots, M\right\}$ and $\left\{q_{i}, i=1, \ldots, M\right\}$ are two normalized probability distributions. We have,

$$
\begin{aligned}
\sum_{j} & \left\{e_{j}\left(\sum_{i} p_{i} \omega_{1 i}\right) e_{j}\left(\sum_{i} q_{i} \omega_{2 i}\right)\right\}^{\frac{1}{2}} \geqslant \\
& \sum_{j} \sum_{i}\left\{p_{i} q_{i} e_{j}\left(\omega_{1 i}\right) e_{j}\left(\omega_{2 i}\right\}^{\frac{1}{2}} \geqslant\right. \\
& \sum_{i} \sqrt{p_{i} q_{i}} \mathcal{F}\left[\omega_{1 i}, \omega_{2 i}\right],
\end{aligned}
$$

the first inequality following from Schwartz inequality and the second inequality from the definition of $\mathcal{F}$. Since eq. (6) holds for any measurement $\left\{e_{j}\right\}$, the definition of $\mathcal{F}$ also implies that

$$
\mathcal{F}\left[\sum_{i} p_{i} \omega_{1 i}, \sum_{i} q_{i} \omega_{2 i}\right] \geqslant \sum_{i} \sqrt{p_{i} q_{i}} \mathcal{F}\left[\omega_{1 i}, \omega_{2 i}\right],
$$


meaning that the generalized fidelity measure complies with the strong concavity property.

Almost all the main properties of the quantum fidelity (2) (including invariance under unitary transformation, whose generalization we are going to discuss in the next section) are shared by the general fidelity measure (1) defined in the BBLW family of probabilistic theories. An important property of the quantum fidelity that is not satisfied in the general scenario is given by Uhlmann's theorem, which says that $\mathcal{F}[\rho, \sigma]=\max _{|\psi\rangle,|\phi\rangle}|\langle\psi \mid \phi\rangle|$, where the maximum is taken over all possible pairs of states $|\psi\rangle$ and $|\phi\rangle$ constituting purifications of the two density matrices $\rho$ and $\sigma$, respectively [13]. A purification of a state $\rho$ (not necessarily pure) of a system $S$ is a pure state $|\psi\rangle$ of a larger system (of which $S$ is a subsystem) such that the corresponding marginal state of $S$ is given by $\rho$. Uhlmann's theorem is not generalizable within the BBLW framework because purification is not possible within arbitrary members of the BBLW family of theories. For instance, purification is not possible within classical probabilistic theories.

Conservation of the fidelity under the evolution of closed systems. - The previously discussed consistency relation between measurements and invertible operations has the following consequence. Given a measurement $\left\{e_{i}\right\}$ to be performed on either of a pair of states $\omega_{1,2}$, and an invertible transformation $\Gamma$, there exists another measurement $\left\{\tilde{e}_{i}\right\}$ yielding the same probabilities when performed, respectively, upon either of the states $\Gamma\left(\omega_{1,2}\right)$. Moreover, given any measurement $\left\{e_{i}\right\}$ to be performed on either of the states $\Gamma\left(\omega_{1,2}\right)$, there is another measurement $\left\{e_{i}^{*}\right\}$ exhibiting the same probabilities when performed upon the states $\omega_{1,2}=\Gamma^{(-1)}\left(\Gamma\left(\omega_{1,2}\right)\right)$. Consequently, it follows from the definition (1) of the generalized fidelity that

$$
\mathcal{F}\left[\Gamma\left(\omega_{1}\right), \Gamma\left(\omega_{2}\right)\right]=\mathcal{F}\left[\omega_{1}, \omega_{2}\right] .
$$

This constitutes a generalization of the invariance property of the quantum fidelity measure under unitary transformations [13]. A similar invariance property is satisfied by the Liouville dynamics of classical ensemble probability densities $[21,22]$. A relation like (8), stating the preservation of the distinguishability between states, can be regarded as meaning that information is conserved by the time evolution of a closed physical system [21].

Generalized Zurek's information transfer theorem. - The quantum no-cloning theorem [23] is nowadays regarded as encapsulating one of the most distinctive features of quantum information. The multiple and profound implications of this principle, and of its various extensions and generalizations, constitute an active research area [24-28].

A recent generalization of the no-cloning theorem advanced by Zurek sheds new light on the nature of the quantum mechanical measurement process [27]. During such a process, information is transferred from the system being measured to the recording apparatus. An intuitive, basic feature of a physical measurement is that its immediate repetition always yields the same result. As a consequence, if the state of the system immediately after the measurement is $\omega$, this state will be left unperturbed if the same measurement is performed a second time quickly after the first one. This uncontroversial assumption is referred to as the predictability postulate [27]. Zurek showed that two basic postulates of quantum mechanics, i) the representation of quantum states by vectors in the system's Hilbert space and ii) the unitarity of quantum evolutions, together with the predictability postulate, are enough to prove that the allowed outcome states of the system after a quantum measurement are restricted to an orthonormal subset of the system's possible states. This result holds true even for imperfect measurements: No matter how little information about the system's state is transferred to the apparatus (as long as this information is not strictly zero) the only allowed unperturbed outcome states for the system have to be orthonormal. This is a profound and intriguing result. It shows that a basic aspect of quantum measurements that is usually regarded as having or needing the status of an independent postulate can actually be derived from the most basic ingredients of quantum theory. Zurek's results have been deservedly hailed as "dissolving one aspect of quantum weirdness" [28]. We are now going to show that a result similar to Zurek's holds true within the general family of probabilistic theories described by the BBLW framework.

Let us consider a special type of processes that are an extension to general probabilistic theories of the processes studied by Zurek [27]. Suppose that we have a composite system $\mathcal{S} \mathcal{A}$ consisting of a system $\mathcal{S}$ and an "apparatus" $\mathcal{A}$. We shall consider the possibility of implementing transformations that transfer information from the system $\mathcal{S}$ to the "recording apparatus" $\mathcal{A}$. In particular, we want to determine under what circumstances this can occur without altering the initial state of $\mathcal{S}$. We assume that $\mathcal{S}$ starts in a pure state $\omega_{i}$ and the apparatus in an initial reference state $\Sigma_{0}$. The process is represented by an invertible transformation $\Gamma$. This does not imply a loss of generality since, as we already said, we assume that the invertible transformations constitute the fundamental ones for closed systems. We can always consider the system $\mathcal{A}$ to be large enough so that the composite system $\mathcal{S} \mathcal{A}$ can be regarded as closed, and all the transformations affecting it can be regarded as invertible. The main question that we want to address is the following: For which initial pure states $\omega_{i}$ of $\mathcal{S}$ is it possible to perform this operation without altering the state $\omega_{i}$ ? Let us consider two initial states $\omega_{1,2}$ for which the aforementioned information transfer is doable. We then have,

$$
\begin{aligned}
& \Gamma\left(\omega_{1} \otimes \Sigma_{0}\right)=\omega_{1} \otimes \Sigma_{1}, \\
& \Gamma\left(\omega_{2} \otimes \Sigma_{0}\right)=\omega_{2} \otimes \Sigma_{2} .
\end{aligned}
$$


Therefore,

$$
\begin{aligned}
\mathcal{F}\left[\omega_{1}, \omega_{2}\right] & =\mathcal{F}\left[\omega_{1} \otimes \Sigma_{0}, \omega_{2} \otimes \Sigma_{0}\right] \\
& =\mathcal{F}\left[\omega_{1} \otimes \Sigma_{1}, \omega_{2} \otimes \Sigma_{2}\right] \\
& \leqslant \mathcal{F}\left[\omega_{1}, \omega_{2}\right] \mathcal{F}\left[\Sigma_{1}, \Sigma_{2}\right]
\end{aligned}
$$

Now, it follows from the last equation that it is not possible to have $\mathcal{F}\left[\Sigma_{1}, \Sigma_{2}\right]<1$ when $\mathcal{F}\left[\omega_{1}, \omega_{2}\right] \neq 0$. Consequently,

$$
\Sigma_{1} \neq \Sigma_{2} \Longrightarrow \mathcal{F}\left[\omega_{1}, \omega_{2}\right]=0 .
$$

That is, if the final states of $\mathcal{A}$ are even partially distinguishable we necessarily have $\mathcal{F}\left[\omega_{1}, \omega_{2}\right]=0$. This means that information can be transferred from system $\mathcal{S}$ to system $\mathcal{A}$ without altering the initial (pure) state of $\mathcal{S}$ only for states of $\mathcal{S}$ that have zero mutual fidelity. That is, if Zurek's process can be implemented for a family $\omega_{i}$ of initial pure states of $\mathcal{S}$, these states must be perfectly distinguishable. This constitutes a generalization of the fundamental theorem recently proved by Zurek $[27,28]$. In terms of the measurement problem our present result can be stated as follows: If a probabilistic theory contained within the BBLW framework complies with the predictability postulate, then the only possible output states of a measurement (even an imperfect one) are restricted to a set of states with mutual zero fidelity. Our generalization of Zurek's theorem constitutes a general principle constraining the transfer of information between physical systems that admits as a particular case the BBLW-no-cloning theorem discussed in [29]. Our approach is different from the one adopted in [29], which is not based on the generalized fidelity measure. Note that in classical probabilistic theories all "pure" states are perfectly distinguishable from each other so that, in such a context, the present result imposes no restrictions on the possible outcomes of measurements.

Generalized no-deleting theorem. - Let us consider now a counterpart of the quantum no-deleting theorem [30] holding within general BBLW probabilistic theories. We are going to study processes whose aim is to delete information from a given system against that of a second system (both initially in the same, unknown state $\omega$ ) bringing the state of one of these systems to a blank state $\omega_{0}$ without altering the state of the other one, i.e., $\omega \otimes \omega \rightarrow \omega \otimes \omega_{0}$. In order to investigate the possible implementation of such a process in a universal way (that is, for any state $\omega$ ) let us consider a tripartite system comprising a source system, a target system, and a "deleting machine". We assume that this tripartite system can be treated as closed, and thus consequently evolves in a reversible way. We shall also assume that initially the source and the target systems are both described by the same state $\omega$ while the deleting machine starts in an initial standard state $\Sigma_{0}$. During the deleting process the information stored in the target system is deleted against that of the source system. Hence, the joint evolution of the tripartite system (which, as said, we assume to be a closed one) is

$$
\Gamma\left(\omega \otimes \omega \otimes \Sigma_{0}\right)=\omega \otimes \omega_{0} \otimes \Sigma_{\omega} .
$$

As already said, we are interested in a universal deleting process. That is, eq. (12) must hold for an arbitrary state $\omega$. In particular, let us assume that (12) is verified for two particular states $\omega_{1,2}$. Then, the conservation of the fidelity measure during the transformation (12) leads to

$$
\begin{aligned}
\mathcal{F} & {\left[\omega_{1} \otimes \omega_{1} \otimes \Sigma_{0}, \omega_{2} \otimes \omega_{2} \otimes \Sigma_{0}\right]=} \\
& \mathcal{F}\left[\Gamma\left(\omega_{1} \otimes \omega_{1} \otimes \Sigma_{0}\right), \Gamma\left(\omega_{2} \otimes \omega_{2} \otimes \Sigma_{0}\right)\right]= \\
& \mathcal{F}\left[\omega_{1} \otimes \omega_{0} \otimes \Sigma_{\omega_{1}}, \omega_{2} \otimes \omega_{0} \otimes \Sigma_{\omega_{2}}\right],
\end{aligned}
$$

Applying now property (3) of the fidelity measure we obtain,

$$
\mathcal{F}\left[\omega_{1} \otimes \omega_{1}, \omega_{2} \otimes \omega_{2}\right]=\mathcal{F}\left[\omega_{1} \otimes \Sigma_{\omega_{1}}, \omega_{2} \otimes \Sigma_{\omega_{2}}\right],
$$

meaning that the initial degree of distinguishability of the source-target composite coincides with the final degree of distinguishability between the source-deleting machine composite. This implies that the information deleted from the target system is not "destroyed", but is entirely transferred into the final state of the deleting machine. This constitutes a generalization of the quantum no-deleting theorem. It is worth mentioning that, strictly speaking, what we have shown is the impossibility of deleting information in a reversible way. The same observation applies to the standard quantum derivation of the no-deleting theorem [30].

Conclusions. - In the present contribution, we have explored some information-related aspects of the BBLW framework for probabilistic physical theories. We have investigated, within this general setting, the main features of a fidelity measure between pairs of states that reduces, in the case of quantum theory, to the standard fidelity measure between statistical operators. We showed that our generalized fidelity measure complies with all the basic properties (excepting Uhlmann's theorem) satisfied by the quantum fidelity measure for density matrices. All the alluded properties admit direct derivations not involving specific features of standard quantum theory, such as the Hilbert space formalism or the properties of density matrices.

We have used the aforementioned fidelity measure to obtain a generalization to the BBLW family of probabilistic theories of Zurek's recent extension of the quantum no-cloning theorem. We considered two systems $\mathcal{S}$ and $\mathcal{A}$ interacting in such a way that a finite amount of information is transferred from $\mathcal{S}$ to $\mathcal{A}$. No matter how little information about the state of $\mathcal{S}$ is finally stored in $\mathcal{A}$ (as long as it is not strictly zero), the only initial states of $\mathcal{S}$ that are left unchanged by this process are restricted to a set of states with vanishing mutual fidelity.

It would be interesting to apply these results to other specific theories (besides classical probabilistic ones or standard quantum mechanics). An interesting candidate is the recent proposal by Hall [31] of a theory incorporating a consistent classical and quantum mixed dynamics. According to Hall's formalism, mixed classical-quantum physical systems are characterized by joint classicalquantum configurations, and states are described by 18004-p5 
mixed classical-quantum ensembles. Hall provides specific prescriptions for evaluating the expectation values of classical phase space functions, on the one hand, and of quantum Hermitian operators, on the other one. It then seems possible to identify "classical effects" with phase space functions $f$ adopting the value 1 inside a given region $\mathcal{R}$ of the classical phase space and vanishing outside $\mathcal{R}$. The expectation value $\langle f\rangle$ then represents the probability of finding the classical part of the system's configuration within the region $\mathcal{R}$. In a similar fashion, "quantum effects" can be associated with Hermitian operators having eigenvalue 1 for some of their eigenstates and eigenvalue zero for the rest (i.e., these operators are projectors on a subspace of the full Hilbert space associated with the possible quantum configurations of the system).

Some of the ideas advanced here may also be useful for analyzing even theories not belonging to the BBLW framework, such as the toy model proposed by Spekkens [20] to explore the ontic vs. epistemic controversy concerning the meaning of the quantum formalism. Spekkens' model is a probabilistic one where a physical state determines the probabilities of getting different outcomes when measuring physical observables. Therefore, it is then possible to define a fidelity measure between states. It would be interesting to explore which of the properties exhibited by fidelity measures in BBLW theories are also satisfied within Spekkens' model.

$$
* * *
$$

The financial assistance of the National Research Foundation (NRF; South African Agency) toward this research is hereby acknowledged. Opinions expressed and conclusions arrived at are those of the authors and are not necessarily to be attributed to NRF. This work was partially supported by the Projects FQM-2445 and FQM-207 of the Junta de Andalucia (Spain, EU).

\section{REFERENCES}

[1] Zurek W. H. (Editor), Complexity, Entropy, and the Physics of Information (Addison-Wesley, Redwood City Cal.) 1990.

[2] Beck C. and Schlogl F., Thermodynamics of Chaotic Systems (Cambridge University Press, Cambridge) 1993.

[3] Frieden B. R. and Soffer B. H., Phys. Rev. E, 52 (1995) 2274.
[4] Frieden B. R., Physics from Fisher Information (Cambridge University Press, Cambridge) 1998.

[5] Frieden B. R., Science from Fisher Information (Cambridge University Press, Cambridge) 2004.

[6] Leff H. S. and Rex A. F. (Editors), Maxwell's Demons 2: Entropy Classical and Quantum Information (Institute of Physics Publishing, Bristol, Philadelphia) 2003.

[7] Lloyd S., Giovannetti V. and Maccone L., Phys. Rev. Lett., 93 (2004) 100501.

[8] Borras A., Zander C., Plastino A. R., Casas M. and Plastino A., EPL, 81 (2008) 30007.

[9] Henrich M. J., Michel M. and Mahler G., Europhys. Lett., 76 (2006) 1057.

[10] Midodashvili P., EPL, 83 (2008) 50004.

[11] Goyal P., Phys. Rev. A, 78 (2008) 052120.

[12] Hall M. J. W., Phys. Rev. A, 59 (1999) 2602.

[13] Nielsen M. A. and Chuang I. L., Quantum Computation and Quantum Information (Cambridge University Press, Cambridge) 2000.

[14] Barnum H., Barrett J., Leifer M. and Wilce A., Phys. Rev. Lett., 99 (2007) 240501.

[15] Primas H., Chemistry Quantum Mechanics and Reductionism (Springer-Verlag, Berlin) 1983.

[16] Gudder S., Quantum Probability (Academic Press, Orlando) 1988.

[17] Weinberg S., Elementary Particles and the Laws of Physics, edited by Mackenzie R. and Doust P. (Cambridge University Press, Cambridge) 1987.

[18] Will C. M., Theory and Experiment in Gravitational Physics (Cambridge University Press, Cambridge) 1993.

[19] Barrett J., Linden N., Massar S., Pironio S., Popescu S. and Roberts D., Phys. Rev. A, 71 (2005) 022101.

[20] Spekkens R. W., Phys. Rev. A, 75 (2007) 032110.

[21] Plastino A. R. and Daffertshofer A., Phys. Rev. Lett., 93 (2004) 138701.

[22] Yamano T. and Iguchi O., EPL, 83 (2008) 50007.

[23] Wootters W. K. and ZureK W. H., Nature, 299 (1982) 802.

[24] Walker T. A. and Braunstein S., Phys. Rev. Lett., 98 (2007) 080501.

[25] Barrett J., Phys. Rev. A, 75 (2007) 032304.

[26] Kalev A. and Hen I., Phys. Rev. Lett., 100 (2008) 210502.

[27] Zurek W. H., Phys. Rev. A, 76 (2007) 052110.

[28] Lloyd S., Nature, 450 (2007) 1167.

[29] Barnum H., Barrett J., Leifer M. and Wilce A., arXiv:quant-ph/0611295.

[30] Pati A. K. and Braunstein S. L., Nature, 404 (2000) 164.

[31] Hall M. J. W., Phys. Rev. A, 78 (2008) 042104. 